\title{
Notes
}

\section{Docking and Scoring of Quercetin and Quercetin Glycosides against $\alpha$-Amylase Receptor}

\author{
Hyunmyung Kim, Jin-Kwang Kim, Linwoo Kang, Karpjoo Jeong, ${ }^{*}$ and Seunho Jung ${ }^{\dagger, *}$

\begin{abstract}
Department of Advanced Technology Fusion, Konkuk University, Seoul 143-701, Korea. *E-mail: jeongk@konkuk.ac.kr ${ }^{\dagger}$ Department of Bioscience and Biotechnology \& Bio/Molecular Informatics Center, Konkuk University, Seoul 143-701, Korea.*E-mail: shjung@konkuk.ac.kr

Received December 21, 2009, Accepted January 14, 2010
\end{abstract}

Key Words: Flavonoid, Quercetin glycoside, Scoring function

Flavonoids are polyphenolic compounds that usually exist in plants as secondary metabolites. ${ }^{1}$ They show strong antioxidative activity as well as other potential effects, including anti-inflammatory, anti-cancer, and anti-viral roles. ${ }^{2}$ Flavonols constitute a major group within the flavonoids present in several foodstuffs, such as apples, cherries, and other green vegetables. The most commonly occurring flavonols are those with dihydroxylation in the 3' and 4' positions of the B ring. The preferred glycosylation site on the flavonoids is the 3 position (Figure 1). Quercetin is the most abundant bioflavonoid and this compound is mainly present in the glycoside form. The bioavailability of quercetin aglycone and its glycosides are different $^{3}$ because of different physical and chemical properties. The bulky glycoside moiety causes a conformational change of the quercetin backbone ${ }^{4}$ and modifies the accessibility to active site of the receptor protein.

In the present study, the interactions among $\alpha$-amylase and quercetin, its monoglycoside isoquercetin, and its diglycoside rutin were investigated by molecular docking study to predict the binding affinity of flavonoid glycosides to receptors. These three flavonoids are effective inhibitors to $\alpha$-amylase, and the inhibitory mode belongs to a competitive type. ${ }^{5}$ There are two factors that are worth due consideration in the scoring and evaluation of the binding affinity. These are the differences of molecular weight due to carbohydrate moiety and the presence of phenolic backbone structure of flavonoids.

Isoquercetin and rutin are quercetin glycosides that have large
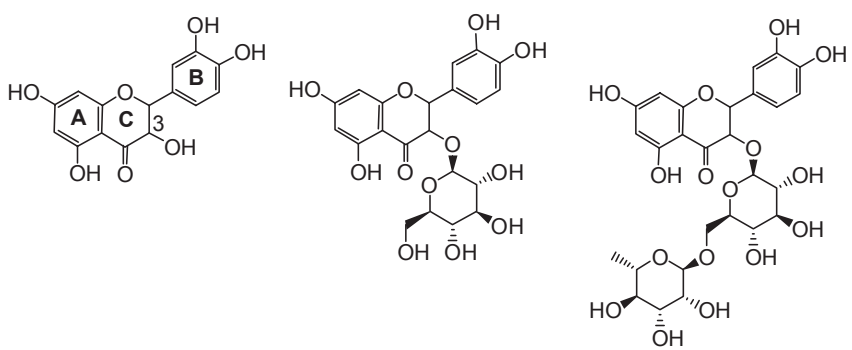

Quercetin (Que) Isoquercetin (Iso)
Rutin (Rut)
Figure 1. Molecular structure of quercetin, isoquercetin, and rutin. bulky carbohydrate moieties of D-glucose and 6-O-L-rhamnosyl-D-glucose, respectively. The molecular weights of quercetin, isoquercetin, and rutin are 302.24, 464.38, and 626.52, respectively (Table 1). Most scoring functions are additive in nature, in the sense that the more functional groups a ligand has, the more interactions it can have with the protein and the greater its calculated intermolecular energy. These scoring functions end up overestimating the binding energy for larger ligands at the expense of smaller ligands. ${ }^{6}$ Therefore, active compounds and decoys were selected with a similar distribution of molecular weight, in order to minimize the effects of the tendency of most scoring functions to favor larger molecules in virtual screening simulations. ${ }^{7}$

Although the carbohydrate moieties of quercetin glycosides give more opportunity to interact with receptors, including hydrogen bond donor/acceptors or interatomic interactions also brings steric hindrance. Steric hindrance is unfavorable to the binding of ligands against receptors. The binding pose of rutin is clearly different from those of quercetin and isoquercetin

Table 1. Scoring functions and other parameters of each flavonoids$\alpha$-amylase complex

\begin{tabular}{cccc}
\hline Scoring Function $^{a}$ & Isoquercetin & Quercetin & Rutin \\
\hline LigScore1 & 5.13 & 4.27 & 5.44 \\
LigScore2 & 5.47 & 5.58 & 5.69 \\
-PLP1 & 117.11 & 88.16 & 138.28 \\
-PLP2 & 121.11 & 86.52 & 132.03 \\
Jain & 2.05 & 0.94 & 1.34 \\
-PMF & 121.22 & 113.04 & 156.03 \\
-PMF04 & 99.22 & 86.03 & 129.41 \\
Ludi_1 & 376 & 339 & 481 \\
Ludi_2 & 321 & 290 & 393 \\
Ludi_3 & 633 & 594 & 529 \\
\hline Others & Isoquercetin & Quercetin & Rutin \\
\hline DOCK_SCORE & 111.17 & 83.44 & 131.49 \\
Molecular Weight & 464.38 & 302.24 & 626.52 \\
\hline
\end{tabular}

\footnotetext{
${ }^{a}$ Averaged one of ten (isoquercetin and quercetin) or nine (rutin) values.
} 


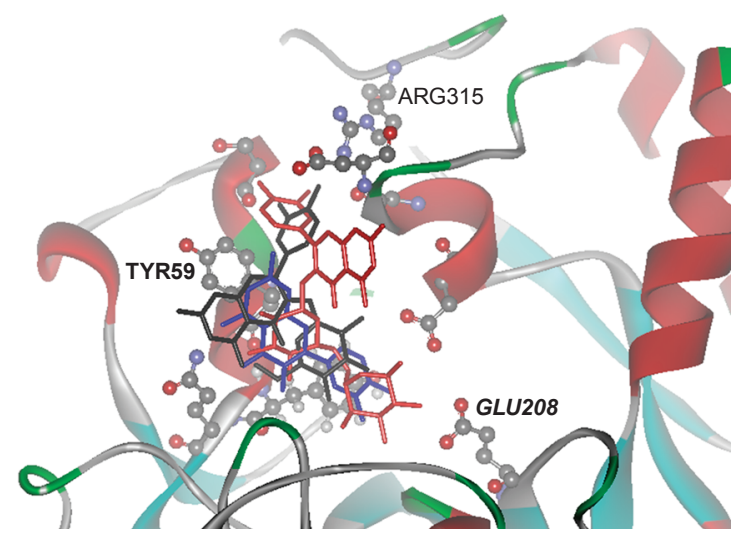

Figure 2. Binding model of three flavonoids against $\alpha$-amylase. Flavonoids and surrounding critical residues are shown as stick and ball \& stick model, respectively. Three flavonoids are colored in black, blue, and red for isoquercetin, quercetin, and rutin, respectively. The detailed interactions between flavonoids and residues of $\alpha$-amylase are described in Figure 3.

(Figure 2). The steric hindrance effect might be more obvious in rutin, which effect is one of the factors of the lower binding affinity and inhibitory activity of rutin compared to those of quercetin and isoquercetin. ${ }^{5}$

There are ten scoring functions in DS LigandFit to improve the scoring efficiency by consensus scoring. ${ }^{8}$ Table 1 shows the results of individual scoring functions. High scores indicate strong flavonoid-ligand binding. Many scoring functions have been developed and optimized to work with docking algorithms. Consensus scoring is applied for predictions of binding poses and binding affinity to obtain better and more consistent results than those studies that used individual scoring functions. The consensus score corresponds to the frequency of occurrence in the top rank percentile of each scoring function. If all scoring functions have comparable precisions, the mean form of several scoring functions should be a better predictor than each individual score. However, in this study, seven scoring functions show the same order of molecular weight of flavonoid ligands (Table 1). The inhibitory and binding orders of three flavonoids against $\alpha$-amylase are Iso $>$ Que $>$ Rut. $^{5}$ Only Ludi_ 3 scoring function shows the same order of binding affinity of flavonoid ligands against $\alpha$-amylase receptor.

LigScore 1 and LigScore2 were calculated from the descriptors of the polar surfaces of the flavonoid-receptor complexes. LigScore1 shows the overestimating of the binding affinity for larger ligands at the expense of smaller ligands. LigScore2 of quercetin is higher than that of isoquercetin, which might be due to the desolvation penalty reconsidered in LigScore2. ${ }^{9}$ However, the larger rutin molecule shows a higher value than that of isoquercetin and is similar to LigScore1. Piecewise linear potential (PLP) scores were calculated based on hydrogen bond formation. The PLP scores of quercetin glycosides are higher than that of quercetin. Potential of mean force (PMF) scores were calculated by summation of pairwise interaction terms of all interatomic pairs in the flavonoid-receptor complex. The PMF scores of the flavonoids show the same pattern as that of LigScore 1 and the PLP scores. The larger flavonoid shows the

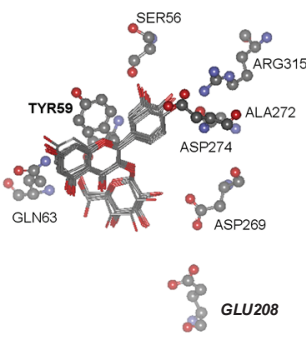

Isoquercetin
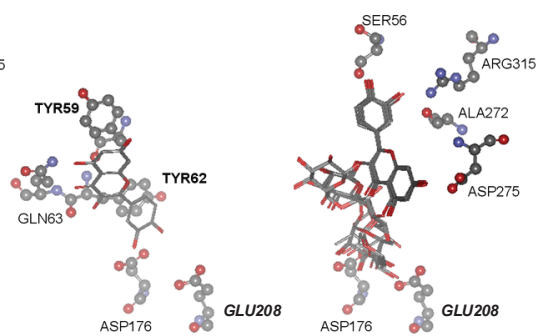

Quercetin

Rutin
Figure 3. The interactions between flavonoids and residues of $\alpha$ amylase nearby are indicated. $\boldsymbol{G L U 2 0 8}$ is a catalytic-site residue of $\alpha$-amylase. Residues with normal letters are hydrogen bonded with each flavonoid. Residues shown in bold are involved in the aromatic interactions ( $\pi-\pi$ interaction; TYR59 and TYR62) with isoquercetin or quercetin flavonoids. Ten posed flavonoids (nine posed in rutin) with highest DOCK SCORE are shown. GLU208 in isoquercetin and quercetin complex have no interaction with the flavonoids and are shown to compare with the rutin complex.

higher score. Jain and Ludi scores are the sum of the contributions such as hydrophobic interaction, polar interaction, entropy and degree of freedom in order to ensure the binding stability in the flavonoid-receptor complex. Jain scoring function predicts that isoquercetin will show the highest binding affinity but underestimates the binding affinity of quercetin against $\alpha$-amylase. Ludi_1 and Ludi_2 scores show the same order of LigScore1 and knowledge-based scoring functions (PLP and PMF). Only Ludi_3 score matches the binding affinity of the flavonoids against $\alpha$-amylase well. Ludi_1 was derived by empirically fitting a set of protein-ligand complexes with experimentally measured binding affinities. ${ }^{10}$ Ludi_2 was subsequently determined by including additional complexes and refitting the weights associated with the above terms. ${ }^{11}$ Ludi_ 3 was determined with the same set of complexes used for Ludi_2, including an additional contribution in the function to account for aromatic-aromatic interactions, ${ }^{11}$ which function improved the scoring of quercetin and quercetin glycosides against $\alpha$ amylase.

Figure 3 shows the binding models of ten flavonoids ( 9 rutin in rutin- $\alpha$-amylase complex) against $\alpha$-amylase. The amino acid residues that participated in hydrogen bonding and $\pi-\pi$ interactions with flavonoid backbone are shown. The common interaction residues of isoquercetin and quercetin complex are TYR59 and GLN63 through $\pi-\pi$ interaction and hydrogen bond, respectively. Isoquercetin and quercetin have no direct interaction with $\boldsymbol{G L U 2 0 8}$, one of the catalytic-site residues of $\alpha$ amylase. TYR59 interacts with A, B, and C rings of isoquercetin and with $A$ and $C$ rings of quercetin, through $\pi-\pi$ interaction (aromatic-aromatic interaction). Other residues are involved in hydrogen bonds, except TYR 62 in quercetin complex, which is involved in $\pi-\pi$ interaction with B ring of quercetin. With similar $\pi$ interactions in these two complexes, isoquercetin has more hydrogen bonding partners than quercetin does, which leads to a higher value for the Ludi_ 3 score. In the case of the rutin- $\alpha$-amylase complex, there is the same number of residues involved in hydrogen bonding with rutin compared with the 
isoquercetin- $\alpha$-amylase complex. It is observed that there is no $\pi-\pi$ interaction between rutin and the residues of $\alpha$-amylase, which leads to a lower value for the Ludi 3 score than that of the other two flavonoid complexes.

The thermodynamic parameters reveal that the main driving force of flavonoid binding is hydrophobic. ${ }^{5}$ This is mainly due to the flavonoid backbone structure and the hydrophobic tubby catalytic center of $\alpha$-amylase. Therefore, the scoring function should be concerned with this hydrophobic interaction in the flavonoid-enzyme system. Ludi_3 scoring function improves this hydrophobic interaction by adding the parameter of aromatic-aromatic interaction. It is recommended to use the Ludi_3 scoring function to predict the rank order of binding affinity of flavonoids, including that of glycosides against receptors. Detailed investigation of the wider set of flavonoid-receptor complexes by experimental and computational methods will be necessary to improve the screening of polyphenolic compounds as drug candidates. Ultimately, consensus scoring with more adequate scoring functions can predict more accurately the binding affinity of flavonoids to various receptors.

\section{Computational Methods}

Molecular docking simulations were performed with the Discovery Studio/LigandFit program (version 2.5, Accelrys Software Inc.) using a PLP. The receptor amylase structure was obtained from the crystallographic geometry of the Protein Data Bank (PDB id 1UA7). The series of flavonoids was constructed and energy-minimized using the Builder module. The binding site was constructed from the grid for the binding cavity of the crystal structure of amylase. This binding site is similar to the binding site of maltopentaose, natural substrate, and acabose, an inhibitor of amylase. ${ }^{12}$ A series of three docking simulations for the receptor amylase with quercetin, isoquercetin, or rutin, was performed on the reconstructed binding site of the receptor protein. The docking of the flavonoids was carried out using the LigandFit module of Discovery Studio. ${ }^{13}$ A PLP was used as an energy grid for docking. Ten scoring functions, LiScore1, LigScore2, PLP1, PLP2, Jain, PMF, PMF04, Ludi1, Ludi2, and Ludi3 were used for scoring. A $10 \AA$ A cutoff was imposed on the calculation of non-bonded interactions, and distance-dependent dielectric constant $(\varepsilon=1 r)$ was used to mimic solvent screening during the conformational searches. The energy grid extension was settled at $3 \AA$; softened potential energy option was used. Conformational search of the Monte Carlo (MC) docking was performed. ${ }^{14}$ An energy tolerance of $10,000 \mathrm{kcal} / \mathrm{mol}$ was imposed to avoid significant overlap of van der Waals radii in the random search. Docked poses within pre-existing clusters were discarded to avoid accepting similar poses. From the MC docking simulations, we analyzed ten docked poses in isoquercetin and quercetin and nine docked poses in rutin with lowest DOCK_SCORE for each flavonoid with $\alpha$-amylase.

Acknowledgments. This study was supported by a grant of Korea Research Foundation (KRF-2006-005-J03402) and partly by Basic Science Research Program through the National Research Foundation of Korea (NRF) funded by the Ministry of Education, Science and Technology (2009-0059986). SDG

\section{References}

1. Havsteen, B. H. Pharmacol. Therapeut. 2002, 96, 67.

2. Rice-Evans, C. A.; Miller, N. J.; Paganga, G. Free Radic. Biol. Med. 1996, 20, 933.

3. Morand, C.; Manach, C.; Crespy, V.; Rémésy, C. Free Radic. Res. 2000, 33, 667.

4. Kim, H.; Jeong, K.; Jung, S. Bull. Korean Chem. Soc. 2006, 27, 325.

5. Li, Y.; Gao, F.; Gao, F.; Shan, F.; Bian, J.; Zhao, C. J. Food Sci. 2009, 74, C199.

6. Kitchen, D. B.; Decornez, H.; Furr, J. R.; Bajorath, J. Nat. Rev. Drug Discov. 2004, 3, 935.

7. Perola, E.; Walters, W. P.; Charifson, P. S. Proteins 2004, 56, 235.

8. Feher, M. Drug Discov. Today 2006, 11, 421.

9. Krammer, A.; Kirchhoff, P. D.; Jiang, X.; Venkatachalam, C. M.; Waldman, M. J. Mol. Graph. 2005, 23, 395.

10. Böhm, H. J. J. Comput. -Aided Mol. Des. 1994, 8, 243.

11. Böhm, H. J. J. Comput. -Aided Mol. Des. 1998, 12, 309.

12. Fujimoto, Z.; Takase, K.; Doui, N.; Momma, M.; Matsumoto, T.; Mizuno, H. J. Mol. Biol. 1998, 277, 393.

13. Choi, Y.; Park, S.; Jeong, K.; Jung, S. Bull. Korean Chem. Soc. 2007, 28, 1811 .

14. Kim, H.; Choi, Y.; Kim, J.-I.; Jeong, K.; Jung, S. Bull. Korean Chem. Soc. 2009, 30, 1373. 